\title{
A Formula for the Logarithm of the KZ Associator ${ }^{\star}$
}

\author{
Benjamin ENRIQUEZ ${ }^{\dagger}$ and Fabio GAVARINI ${ }^{\ddagger}$ \\ † IRMA (CNRS), rue René Descartes, F-67084 Strasbourg, France \\ E-mail: enriquez@math.u-strasbg.fr \\ $\ddagger$ Universitá degli Studi di Roma "Tor Vergata", Dipartimento di Matematica, \\ Via della Ricerca Scientif ica 1, I-00133 Rome, Italy \\ E-mail: gavarini@mat.uniroma2.it
}

Received October 03, 2006, in final form November 10, 2006; Published online November 13, 2006 Original article is available at http://www.emis.de/journals/SIGMA/2006/Paper080/

\begin{abstract}
We prove that the logarithm of a group-like element in a free algebra coincides with its image by a certain linear map. We use this result and the formula of Le and Murakami for the Knizhnik-Zamolodchikov (KZ) associator $\Phi$ to derive a formula for $\log (\Phi)$ in terms of MZV's (multiple zeta values).
\end{abstract}

Key words: free Lie algebras; Campbell-Baker-Hausdorff series, Knizhnik-Zamolodchikov associator

2000 Mathematics Subject Classification: 17B01; 81R50

To the memory of Vadim Kuznetsov.

\section{Logarithms of group-like elements}

Let $F_{n}$ be the free associative algebra generated by free variables $x_{1}, \ldots, x_{n}$, let $\mathfrak{f}_{n} \subset F_{n}$ be the free Lie algebra with the same generators, and let $\widehat{\mathfrak{f}}_{n}, \widehat{F}_{n}$ be their degree completions (where $x_{1}, \ldots, x_{n}$ have degree 1$)$. A group-like element of $\widehat{F}_{n}$ is an element of the form $X=1+($ terms of degree $>0$ ), such that $\Delta(X)=X \otimes X$, where $\Delta$ is the completion of the coproduct $F_{n} \rightarrow F_{n}^{\otimes 2}$, for which $x_{1}, \ldots, x_{n}$ are primitive. It is well-known that the exponential defines a bijection exp: $\widehat{\mathfrak{f}}_{n} \rightarrow$ group-like elements of $\widehat{F}_{n}$ \} (also denoted $x \mapsto e^{x}$ ). We denote by log the inverse bijection.

We denote by $\mathrm{CBH}_{n}\left(x_{1}, \ldots, x_{n}\right)$ the multilinear part (in $\left.x_{1}, \ldots, x_{n}\right)$ of $\log \left(e^{x_{1}} \cdots e^{x_{n}}\right)$. Define a linear map

$$
\operatorname{cbh}_{n}: F_{n} \rightarrow \mathfrak{f}_{n}
$$

by $\operatorname{cbh}_{n}(1)=0$ and $\operatorname{cbh}_{n}\left(x_{i_{1}} \cdots x_{i_{k}}\right):=\mathrm{CBH}_{k}\left(x_{i_{1}}, \ldots, x_{i_{k}}\right)$. This map extends to a linear map $\widehat{\operatorname{cbh}}_{n}: \widehat{F}_{n} \rightarrow \widehat{\mathfrak{f}}_{n}$.

Proposition 1. If $X \in \widehat{F}_{n}$ is group-like, then $\log (X)=\widehat{\operatorname{cbh}}_{n}(X)$.

Proof. It is known that $F_{n}=U\left(\mathfrak{f}_{n}\right)$, so that the symmetrization is an isomorphism sym: $S\left(\mathfrak{f}_{n}\right) \rightarrow F_{n}$. Denote by $p_{n}: F_{n} \rightarrow \mathfrak{f}_{n}$ the composition of $\operatorname{sym}^{-1}$ with the projection $S\left(\mathfrak{f}_{n}\right)=\oplus_{k \geq 0} S^{k}\left(\mathfrak{f}_{n}\right)$ onto $S^{1}\left(\mathfrak{f}_{n}\right)=\mathfrak{f}_{n}$. We first prove:

Lemma 1. $p_{n}=\operatorname{cbh}_{n}$.

\footnotetext{
${ }^{\star}$ This paper is a contribution to the Vadim Kuznetsov Memorial Issue "Integrable Systems and Related Topics". The full collection is available at http://www.emis.de/journals/SIGMA/kuznetsov.html
} 
Proof. If $\mathfrak{g}$ is a Lie algebra, let $p_{\mathfrak{g}}: U(\mathfrak{g}) \rightarrow \mathfrak{g}$ be the composition of the inverse of the symmetrization $S(\mathfrak{g}) \rightarrow U(\mathfrak{g})$ with the projection onto the first component of $S(\mathfrak{g})$. If $\phi: \mathfrak{g} \rightarrow \mathfrak{h}$ is a Lie algebra morphism, then we have a commutative diagram

$\begin{array}{lll}U(\mathfrak{g}) & \stackrel{p_{\mathfrak{g}}}{\longrightarrow} & \mathfrak{g} \\ U(\phi) \downarrow & & \downarrow \phi \\ U(\mathfrak{h}) & \stackrel{p_{\mathfrak{h}}}{\longrightarrow} & \mathfrak{h}\end{array}$

It follows from Lemma 3.10 of [4] that if $k \geq 0$ and $F_{k}$ is the free algebra with generators $y_{1}, \ldots, y_{k}$, then $p_{k}\left(y_{1}, \ldots, y_{k}\right)=\mathrm{CBH}_{k}\left(y_{1}, \ldots, y_{k}\right)$. If now $\boldsymbol{i}=\left(i_{1}, \ldots, i_{k}\right)$ is a sequence of elements of $\{1, \ldots, n\}$, we have a unique morphism $\phi_{i}: \mathfrak{f}_{k} \rightarrow \mathfrak{f}_{n}$, such that $y_{1} \mapsto x_{i_{1}}, \ldots$, $y_{k} \mapsto x_{i_{k}}$.

Then

$$
\begin{aligned}
p_{n}\left(x_{i_{1}} \cdots x_{i_{k}}\right) & =p_{\mathfrak{f}_{n}} \circ U\left(\phi_{\boldsymbol{i}}\right)\left(y_{1} \cdots y_{k}\right)=\phi_{\boldsymbol{i}} \circ p_{\mathfrak{f}_{k}}\left(y_{1} \cdots y_{k}\right) \\
& =\phi_{\boldsymbol{i}}\left(\mathrm{CBH}_{k}\left(y_{1}, \ldots, y_{k}\right)\right)=\mathrm{CBH}_{k}\left(x_{i_{1}}, \ldots, x_{i_{k}}\right)=\operatorname{cbh}_{n}\left(x_{i_{1}} \cdots x_{i_{k}}\right),
\end{aligned}
$$

which proves the lemma.

End of proof of Proposition 1. We denote by $\widehat{p}_{n}: \widehat{F}_{n} \rightarrow \widehat{\mathfrak{f}}_{n}$ the map similarly derived from the isomorphism $\widehat{F}_{n} \simeq \widehat{\oplus}_{k \geq 0} S^{k}\left(\widehat{\mathfrak{f}}_{n}\right)$ (where $\widehat{\oplus}$ is the direct product). Then $p_{n}=\operatorname{cbh}_{n}$ implies $\widehat{p}_{n}=\widehat{\operatorname{cbh}}_{n}$.

If now $X \in \widehat{F}_{n}$ is group-like, let $\ell:=\log (X)$. We have $X=1+\ell+\ell^{2} / 2 !+\cdots ;$ here $\ell^{k} \in S^{k}\left(\widehat{\mathfrak{f}}_{n}\right)$, so $\widehat{p}_{n}(X)=\ell$. Hence $\widehat{\operatorname{cbh}}_{n}(X)=\ell=\log (X)$.

\section{Corollaries}

The $\mathrm{KZ}$ associator is defined as follows. Let $A_{0}, A_{1}$ be noncommutative variables. Let $F_{2}$ be the free associative algebra generated by $A_{0}$ and $A_{1}$, let $\mathfrak{f}_{2} \subset F_{2}$ be its (free) Lie subalgebra generated by $A_{0}$ and $A_{1}$. Let $\widehat{F}_{2}$ and $\widehat{\mathfrak{f}}_{2}$ be the degree completions of $F_{2}$ and $\mathfrak{f}_{2}\left(A_{0}\right.$ and $A_{1}$ have degree 1).

The KZ associator $\Phi$ is defined [1] as the renormalized holonomy from 0 to 1 of the differential equation

$$
G^{\prime}(z)=\left(\frac{A_{0}}{z}+\frac{A_{1}}{z-1}\right) G(z)
$$

i.e., $\Phi=G_{1} G_{0}^{-1}$, where $G_{0}, G_{1} \in \widehat{F}_{2} \widehat{\otimes} \mathcal{O}_{] 0,1[}$ are the solutions of (1) with $G_{0}(z) \sim z^{A_{0}}$ as $z \rightarrow 0^{+}$ and $G_{1}(z) \sim(1-z)^{A_{1}}$ as $z \rightarrow 1^{-}$; here $\mathcal{O}_{] 0,1[}$ is the ring of analytic functions on $] 0,1[$, and $\widehat{F}_{2} \widehat{\otimes} V$ is the completion of $F_{2} \otimes V$ w.r.t. the topology defined by the $F_{2}^{\geq n} \otimes V$ (here $F_{2}^{\geq n}$ is the part of $F_{2}$ of degree $\geq n$ ).

We recall Le and Murakami's formula for $\Phi[3]$. We say that a sequence $\left(a_{1}, \ldots, a_{n}\right) \in\{0,1\}^{n}$ is admissible if $a_{1}=1$ and $a_{n}=0$. If $\left(a_{1}, \ldots, a_{n}\right)$ is admissible, we set

$$
\omega_{a_{1}, \ldots, a_{n}}=\int_{0}^{1} \omega_{a_{1}} \circ \cdots \circ \omega_{a_{n}}
$$

where $\omega_{0}(t)=d t / t, \omega_{1}(t)=d t /(t-1)$ and $\int_{a}^{b} \alpha_{1} \circ \cdots \circ \alpha_{n}=\int_{a \leq t_{1} \leq \cdots \leq t_{n} \leq b} \alpha_{1}\left(t_{1}\right) \wedge \cdots \wedge \alpha_{n}\left(t_{n}\right)$. Up to sign, the $\omega_{a_{1}, \ldots, a_{n}}$ are MZV's (multiple zeta values). 
If $\left(i_{1}, \ldots, i_{n}\right)$ is an arbitrary sequence in $\{0,1\}^{n}$, and $\left(a_{1}, \ldots, a_{n}\right)$ is an admissible sequence, define integers $C_{i_{1}, \ldots, i_{n}}^{a_{1}, \ldots, a_{n}}$ by the relation

$$
\begin{aligned}
& \sum_{\left(i_{1}, \ldots, i_{n}\right) \in\{0,1\}^{n}} C_{i_{1}, \ldots, i_{n}}^{a_{1}, \ldots, a_{n}} A_{i_{n}} \cdots A_{i_{1}} \\
& =\sum_{\substack{S \subset\left\{\alpha \mid a_{\alpha}=0\right\}, T \subset\left\{\beta \mid a_{\beta}=1\right\}}}(-1)^{\operatorname{card}(S)+\operatorname{card}(T)} A_{1}^{\operatorname{card}(T)} A\left(a_{1}, \ldots, a_{n}\right)^{S, T} A_{0}^{\operatorname{card}(S)},
\end{aligned}
$$

where for any $S \subset\left\{\alpha \mid a_{\alpha}=0\right\}, T \subset\left\{\beta \mid a_{\beta}=1\right\}, A\left(a_{1}, \ldots, a_{n}\right)^{S, T}:=\prod_{\alpha \in[1, n] \backslash(S \cup T)} A_{a_{\alpha}}$ (the product is taken in decreasing order of the $\alpha$ 's).

Theorem 1 ([3]).

$$
\Phi=1+\sum_{n \geq 1} \sum_{\substack{\left(a_{1}, \ldots, a_{n}\right) \text { admissible } \\\left(i_{1}, \ldots, i_{n}\right) \in\{0,1\}^{n}}} \omega_{a_{1}, \ldots, a_{n}} C_{i_{1}, \ldots, i_{n}}^{a_{1}, \ldots, a_{n}} A_{i_{n}} \cdots A_{i_{1}} .
$$

Since $\Phi \in \widehat{F}_{2}$ is a group-like element, Proposition 1 implies that $\log (\Phi)=\widehat{\operatorname{cbh}}_{2}(\Phi)$, therefore:

\section{Corollary 1.}

$$
\log (\Phi)=\sum_{n \geq 1} \sum_{\substack{\left(a_{1}, \ldots, a_{n}\right) \text { admissible } \\\left(i_{1}, \ldots, i_{n}\right) \in\{0,1\}^{n}}} \omega_{a_{1}, \ldots, a_{n}} C_{i_{1}, \ldots, i_{n}}^{a_{1}, \ldots, a_{n}} \mathrm{CBH}_{n}\left(A_{i_{n}}, \ldots, A_{i_{1}}\right) .
$$

Using the explicit formula of [2], one computes similarly the logarithm of the analogue $\Psi$ of the $\mathrm{KZ}$ associator of the equation $G^{\prime}(z)=\left(A / z+\sum_{\zeta \mid \zeta^{n}=1} b_{\zeta} /(z-\zeta)\right) G(z)$.

Proposition 1 also implies:

Lemma 2. Let $\mathfrak{g}$ be a nilpotent Lie algebra, $G$ be the associated Lie group, let $a<b \in \mathbb{R}$. Fix $h(z) \in C^{0}([a, b], \mathfrak{g})$ and let $H$ be the holonomy from a to $b$ of the differential equation $H^{\prime}(z)=h(z) H(z)$, where $H(z) \in C^{1}([a, b], G)$. Then

$$
\log (H)=\sum_{n \geq 1} \int_{a \leq z_{1} \leq \cdots \leq z_{n} \leq b} \operatorname{CBH}_{n}\left(h\left(z_{n}\right), \ldots, h\left(z_{1}\right)\right) d z_{1} \cdots d z_{n} .
$$

\section{Acknowledgements}

We first established the formula for $\log (\Phi)$ in Corollary 1 by analytic computations (using a direct proof of Lemma 2). It was the referee who remarked its formal similarity with the formula of Le and Murakami (Theorem 1); this remark can be expressed as the equality $\log (\Phi)=\widehat{\operatorname{cbh}}_{2}(\Phi)$. This led us to try and understand whether this formula followed from the group-likeness of $\Phi$, which is indeed the case (Proposition 1). C. Reutenauer then pointed out that a part of our argument is a result in his book.

[1] Drinfeld V., On quasitriangular quasi-Hopf algebras and a group closely connected with $\operatorname{Gal}(\overline{\mathbb{Q}} / \mathbb{Q})$, Leningrad Math. J., 1991, V.2, 829-860.

[2] Enriquez B., Quasi-reflection algebras, multiple polylogarithms at roots of 1, and analogues of the group GT, math.QA/0408035.

[3] Le T.T.Q., Murakami J., Kontsevich's integral for the Kauffman polynomial, Nagoya Math. J., 1996, V.142, 39-65.

[4] Reutenauer C., Free Lie algebras, London Mathematical Society Monographs. New Series, Vol. 7, Oxford Science Publications, New York, The Clarendon Press, Oxford University Press, 1993. 